\title{
Incidência das mulheres submetidas à curetagem uterina pós-abortamento no serviço de saúde pública
}

\author{
Incidence of women submitted to post-abortion uterine cureting in the public health service \\ Incidencia de mujeres sujetas a curetación uterina posterior al aborto en el servicio de salud \\ pública
}

Recebido: 12/01/2021 | Revisado: 20/01/2021 | Aceito: 21/01/2021 | Publicado: 25/01/2021

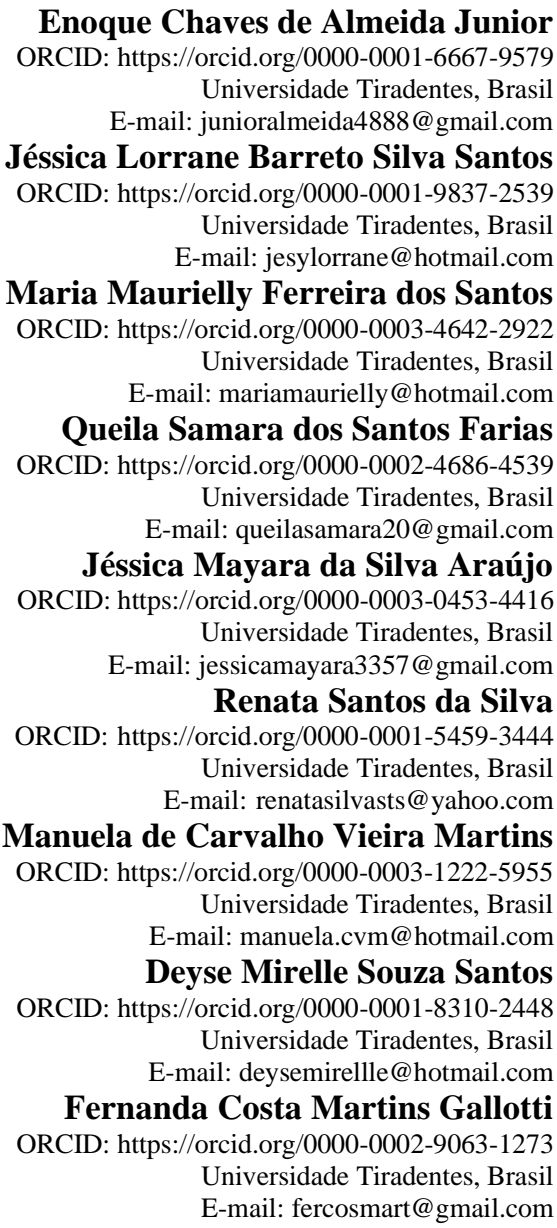

\section{Resumo}

A curetagem uterina é um dos procedimentos mais realizados na rede pública de saúde, principalmente no pósabortamento, para a extração do produto conceptual deixado na cavidade uterina. Para tanto, buscou-se neste artigo analisar o quantitativo e o total de dias de permanência hospitalar das mulheres que se submeteram à curetagem uterina após abortamento no Sistema Único de Saúde. Trata-se de um estudo ecológico de natureza básica, retrospectivo com abordagem quantitativa de caráter descritivo, realizado por meio de consulta ao Departamento de Informática do Sistema Único de Saúde (DATASUS). Foram observados os números de abortos e curetagens no pósabortamento entre 2017 a 2019 nos municípios do Estado de Sergipe. Os dados obtidos foram reorganizados e analisados por meio do programa Excel versão 2010, apresentados em tabelas com números absolutos e porcentagens. Após análise dos dados, notou-se que nos anos de 2017, 2018 e 2019, houve um total de 7.941 casos de curetagem uterina realizadas nas regiões de Sergipe. Desses, 3.196 mulheres sofreram aborto espontâneo e, realizaram esse procedimento, tendo um total de 12.362 dias de permanência hospitalar, resultando gasto médio de R $\$ 684,75$. Desse modo, a curetagem uterina é a terapêutica mais utilizada em casos de abortos no ambiente hospitalar. Por ser um procedimento cirúrgico, necessita de maior tempo de internação, implicando nos altos custos para a saúde pública.

Palavras-chave: Aborto; Curetagem; Saúde da mulher.

\section{Abstract}

Uterine curettage is one of the most performed procedures in the public health system, especially in post-abortion, for 
the extraction of the conceptual product left in the uterine cavity. To this end, this article sought to analyze the quantity and total days of hospital stay of women who underwent uterine curettage after abortion in the Unified Health System. This is an ecological study of a basic, retrospective nature with a quantitative approach of a descriptive character, carried out through consultation with the Informatics Department of the Unified Health System (DATASUS). The number of abortions and curettages in the post-abortion period between 2017 and 2019 were observed in the municipalities of the State of Sergipe. The data obtained were reorganized and analyzed using the Excel version 2010 program, presented in tables with absolute numbers and percentages. After analyzing the data, it was noted that in the years 2017, 2018 and 2019, there were a total of 7,941 cases of uterine curettage performed in the regions of Sergipe. Of these, 3,196 women suffered spontaneous abortion and underwent this procedure, having a total of 12,362 days of hospital stay, resulting in an average expense of $\mathrm{R} \$ 684.75$. Thus, uterine curettage is the most used therapy in cases of abortions in the hospital environment. As it is a surgical procedure, it requires a longer hospital stay, implying high costs for public health.

Keywords: Abortion; Curettage; Women's health.

\section{Resumen}

El legrado uterino es uno de los procedimientos más realizados en el sistema de salud pública, especialmente en el postaborto, para la extracción del producto conceptual que queda en la cavidad uterina. Para ello, este artículo buscó analizar la cantidad y el total de días de estadía hospitalaria de las mujeres que se sometieron a legrado uterino luego de un aborto en el Sistema Único de Salud. Se trata de un estudio ecológico de carácter básico, retrospectivo con abordaje cuantitativo de carácter descriptivo, realizado mediante consulta con el Departamento de Informática del Sistema Único de Salud (DATASUS). Se observó la cantidad de abortos y legrados en el período postaborto entre 2017 y 2019 en los municipios del Estado de Sergipe. Los datos obtenidos fueron reorganizados y analizados mediante el programa Excel versión 2010, presentados en tablas con números absolutos y porcentajes. Tras analizar los datos, se constató que en los años 2017, 2018 y 2019, se realizaron un total de 7,941 casos de legrado uterino realizado en las regiones de Sergipe. De ellas, 3.196 mujeres sufrieron aborto espontáneo y se sometieron a este procedimiento, con un total de 12.362 días de estancia hospitalaria, lo que resultó en un gasto promedio de $\mathrm{R} \$ 684,75$. Así, el legrado uterino es la terapia más utilizada en casos de abortos en el ámbito hospitalario. Al tratarse de un procedimiento quirúrgico, requiere una mayor estancia hospitalaria, lo que implica altos costos para la salud pública.

Palabras-clave: Aborto; Legrado; La Salud de la mujer.

\section{Introdução}

O aborto é uma temática considerada como um importante problema de saúde pública em países subdesenvolvidos, como o Brasil, tendo em vista que envolve aspectos jurídicos, éticos, legais, médicos e culturais (Chaves \& Pereira, 2020; Leila, Silva, Bonan \& Fonseca, 2015). Tal ato tem grande repercussão na vida da mulher, pois, tende a alterar alguns funcionamentos no seu corpo, tanto fisiológico quanto psicológico, especialmente quando a mesma não espera determinada ocorrência (Silva, Sales \& Albuquerque, 2020).

Segundo os dados apresentados pelo Ministério da Saúde, estima-se que o aborto espontâneo resulta entre 10 a $15 \%$ das gestações, e traz sentimentos de luto, perda e culpa para a mulher por não conseguir manter a gestação (Leila et al., 2015; Brasil 2011; Domingues, Fonseca, Aquino \& Menezes, 2020). Esses dados podem ser reduzidos se as gestantes tiverem uma assistência obstétrica qualificada durante o pré-natal, repercutindo positivamente no período gravídico-puerperal (Adesse, Jannotti, Silva \& Fonseca, 2016; Ribeiro, Albuquerque \& Souza, 2017).

O aborto é um tema polêmico, o qual cabe à mulher decidir se deseja ser mãe em alguma fase de sua vida (Lowy, 2020). De acordo com a medicina, o abortamento é definido como a interrupção da gravidez até a $22^{a}$ semana de gestação, com feto pesando menos que $500 \mathrm{~g}$ ou antes de 20 semanas completas de idade gestacional, não possuindo nenhuma probabilidade de sobrevida, sendo que o aborto é o produto eliminado pelo abortamento (Araújo, Dornelas \& Sousa, 2018).

As causas mais comuns dos abortos espontâneos envolvem, dentre outros, a idade materna avançada, multiplicidade de parceiros e, com isso, estima-se que mulheres com 35 anos apresentam $20 \%$ de chances de abortamento, aos 40 o índice sobe para $40 \%$ e aos 45 anos a probabilidade aumenta para 80\%. Há também outros fatores que podem contribuir para a interrupção da gravidez como: tabagismo, baixo nível de escolaridade e histórico de aborto prévio (França, Sakae \& Kleveston, 2018; Domingues et al., 2020). 
No Brasil, o ato de interromper a gestação é considerado crime. No entanto, segundo o Código Penal Brasileiro (artigos 124-127), há duas situações em que é possível realizá-lo: quando não existe outro meio para salvar a vida da gestante ou quando a gravidez resulte de estupro, descrito no artigo 128 (Decreto n. 2.848, 1940). Apesar de ser ilegal, não tem impedido que ocorra de forma disseminada e insegura entre as diferentes classes sociais no país, sobretudo, nas regiões menos desenvolvidas e por mulheres vulneráveis socialmente (Domingues et al., 2020).

Nesses casos, de acordo com Brasil (2011), em gestações inferiores a doze semanas deve-se avaliar o tamanho do útero e, a partir disso, indicar a melhor técnica para a remoção do feto ou restos placentários. Geralmente, realiza-se a aspiração manual ou a elétrica intrauterina e, quando não for possível realizá-la é feito o procedimento de curetagem uterina nos serviços de saúde pública. Em gestações com menos de doze semanas, pode-se utilizar o misoprostol, por trazer menos riscos à saúde da mulher, além do baixo preço (Madeiro \& Rufino, 2017).

Apesar de existirem diversas terapêuticas para o tratamento do aborto, a curetagem uterina continua sendo um dos procedimentos obstétricos mais executados na rede pública de saúde, no entanto, quando realizada de forma inapropriada pode levar ao óbito materno, elevando assim, os custos para os cofres públicos. Esse procedimento cirúrgico consiste no esvaziamento da cavidade endometrial por meio de uma raspagem, retirando o material desprendido através da cureta (Brasil, 2011; Cardoso, Vieira \& Saraceni, 2020). Durante sua realização, Araújo (2018) ressalta que o sangramento persistente, hemorragia, endometrite, restos placentários, hematoma de colo uterino e perfuração uterina são possíveis complicações que podem ocorrer durante $\mathrm{o}$ ato.

A escassez de trabalhos científicos atualizados nos bancos de dados e o elevado quantitativo de abortos espontâneos realizados justificam a elaboração do presente artigo como meio de levantar novos dados a respeito da curetagem uterina. Para isso, utilizou-se como questão norteadora: Qual a probabilidade das mulheres que sofreram aborto realizarem curetagem uterina? Com o objetivo de analisar os casos de aborto e o tempo de permanência hospitalar das mulheres que se submeteram à curetagem uterina após abortamento no Sistema Único de Saúde no período de 2017 a 2019 no Estado de Sergipe.

\section{Metodologia}

Trata-se de um estudo ecológico, de natureza quantitativa e caráter descritivo, cuja coleta de dados foi realizada entre março e maio de 2020. Para Pereira et al. (2018), os métodos quantitativos fazem coletas de dados numéricos ou quantitativos, possibilitando através do enfoque matemático, a utilização de métodos estatísticos objetivando realizar previsões de algum fenômeno em estudo na área da saúde.

Os dados secundários foram colhidos mediante busca eletrônica nos registros do SINAN (Sistema de Informação de Agravos de Notificação), disponibilizados pelo Departamento de Informática do Sistema Único de Saúde - DATASUS.

Para o embasamento teórico, utilizou-se também, protocolos e artigos indexados nas bases de dados da Biblioteca Virtual de Saúde (BVS), Scientific Electronic Library Online (Scielo), National Library of Medicine and National Institute of Health - USA (PubMed) e na Literatura Latino-americana e do Caribe em Ciências da Saúde (Lilacs), disponíveis em português e inglês publicados durante o período de 2009 a 2020.

A busca foi realizada no mês de abril de 2020 utilizando os descritores saúde da mulher, aborto e curetagem, separados pelos operadores boleanos AND e OR como estratégia de busca.

A população do estudo foi constituída por todos os casos de mulheres que realizaram a curetagem uterina no pósabortamento espontâneo, durante o período de 2017 a 2019 no Estado de Sergipe, com idade entre 15 a 29 anos. Essa faixa etária é mais vulnerável socialmente, pois está exposta a variações socioeconômicas que refletem no aumento da desigualdade.

Para evitar erros de retardo de notificação, optou-se por analisar os três últimos anos em que constavam os dados completos. Foram excluídos os dados dos procedimentos de curetagem uterina realizados dentro do serviço privado de saúde, 
de mulheres que sofreram aborto e realizaram outros procedimentos independente da idade gestacional.

Os dados foram obtidos em dois momentos no DATASUS. Primeiro, foram utilizadas as variáveis: lista morb CID-10, município, internações hospitalares aprovadas e faixa etária 1, os filtros utilizados foram aborto espontâneo e faixa etária entre 15 a 29 anos. No segundo momento, as variáveis foram município, internações hospitalares aprovadas, procedimento, valor médio de internação e dias de permanência hospitalar, filtrados em curetagem uterina.

Para a análise estatística, foram construídas tabelas e gráficos por meio do programa Microsoft Office Excel versão 2010, calculando-se frequências absolutas e porcentagens para cada variável.

Por se tratar de um banco de domínio público, não foi necessário submeter à pesquisa ao Comitê de Ética em Pesquisa.

\section{Resultados}

Após busca realizada na base de dados DATASUS, foram selecionados dados sobre o aborto espontâneo e curetagem uterina pós-abortamento, realizados nos municípios do Estado de Sergipe conforme os critérios de inclusão e exclusão. Os dados coletados foram representados e estruturados através da seguinte tabela: 
Tabela 1 - Distribuição das variáveis de abortos espontâneos, curetagens uterinas e tempo de internação após abortamento espontâneo. Sergipe, 2017 a 2019.

\begin{tabular}{|c|c|c|c|c|c|c|}
\hline \multirow{2}{*}{$\begin{array}{l}\text { Variáveis } \\
\text { ABORTO ESPONTÂNEO }\end{array}$} & \multicolumn{6}{|c|}{ ANOS } \\
\hline & 2017 & $\%$ & 2018 & $\%$ & 2019 & $\%$ \\
\hline Aracaju & 415 & 38,30 & 404 & 38 & 411 & 39,20 \\
\hline Estância & 153 & 14,10 & 116 & 10,90 & 135 & 12,90 \\
\hline Itabaiana & 9 & 0,80 & 8 & 0,70 & 11 & 1 \\
\hline Lagarto & 146 & 13,50 & 156 & 14,70 & 140 & 13,40 \\
\hline Nossa Senhora da Glória & 42 & 3,80 & 36 & 3,40 & 68 & 6,50 \\
\hline Nossa Senhora do Socorro & 262 & 24,20 & 258 & 24,20 & 197 & 18,80 \\
\hline Propriá & 57 & 5,30 & 86 & 8,10 & 86 & 8,20 \\
\hline TOTAL & 1.084 & 100 & 1.064 & 100 & 1.048 & 100 \\
\hline \multicolumn{7}{|l|}{ CURETAGEM UTERINA } \\
\hline Aracaju & 1.105 & 41,20 & 1.137 & 42,28 & 1.291 & 50,20 \\
\hline Capela & 39 & 1,50 & 57 & 2,12 & 41 & 1,60 \\
\hline Estância & 275 & 10,30 & 154 & 5,73 & 138 & 5,40 \\
\hline Itabaiana & 390 & 14,60 & 423 & 15,73 & 366 & 14,20 \\
\hline Lagarto & 286 & 10,70 & 304 & 11,31 & 271 & 10,50 \\
\hline Nossa Senhora da Glória & 60 & 2,20 & 42 & 1,56 & 101 & 3,90 \\
\hline Nossa Senhora do Socorro & 452 & 16,80 & 447 & 16,62 & 278 & 10,80 \\
\hline Propriá & 72 & 2,70 & 125 & 4,65 & 87 & 3,40 \\
\hline TOTAL & 2.679 & 100 & 2.689 & 100 & 2.573 & 100 \\
\hline \multicolumn{7}{|l|}{ TEMPO DE INTERNAÇÃO } \\
\hline Aracaju & 1.765 & 44,22 & 1.818 & 43 & 2.203 & 53,23 \\
\hline Capela & 40 & 1 & 60 & 1,40 & 49 & 1,18 \\
\hline Estância & 363 & 9,10 & 194 & 4,60 & 178 & 4,30 \\
\hline Itabaiana & 530 & 13,28 & 583 & 13,70 & 553 & 13,36 \\
\hline Lagarto & 422 & 10,58 & 618 & 14,60 & 428 & 10,35 \\
\hline Nossa Senhora da Glória & 73 & 1,82 & 50 & 1,20 & 146 & 3,52 \\
\hline Nossa Senhora do Socorro & 702 & 17,59 & 736 & 17,40 & 433 & 10,46 \\
\hline Propriá & 96 & 2,41 & 173 & 4,10 & 149 & 3,60 \\
\hline TOTAL & 3.991 & 100 & 4.232 & 100 & 4.139 & 100 \\
\hline
\end{tabular}

Fonte: DATASUS (2020).

Na Tabela 1 é possível observar que a ocorrência de abortos espontâneos teve um decréscimo entre os anos observados. Ao analisar os três anos, observou-se que em 2017, obteve 1.084 registros de aborto espontâneo, em 2018 houve 1.064 registros e em 2019 foram 1.048 casos.

Ligado a isso, têm-se o número de curetagens uterinas realizadas no pós-abortamento entre os anos de 2017 a 2019 , totalizando 7.941 casos no Estado de Sergipe. Desses, 3.533 foram no município de Aracaju, registrando um aumento de 10\% entre os anos analisados. Em contrapartida, o município de Capela apresentou apenas 1,50\% dos procedimentos realizados em 2017 e 1,60\% em 2019, uma vez que o município de Nossa Senhora da Glória obteve o menor percentual em 2018 com 1,56\%.

A análise dos dados de internação hospitalar por aborto espontâneo no Estado de Sergipe entre os anos analisados relevou que em 2017, houve um total de 3.991 dias de internação hospitalar, aumentando para 4.232 dias em 2018 . No ano 
seguinte, foram registrados 4.139 dias de internação após aborto espontâneo, havendo assim, uma queda em relação a 2018.

É possível observar a variação dos custos médios por internação pós abortamento no período de 2017 a 2019 (Gráfico $1)$.

Gráfico 1: Distribuição do valor médio de internação pós abortamento espontâneo. Sergipe, 2017 a 2019.

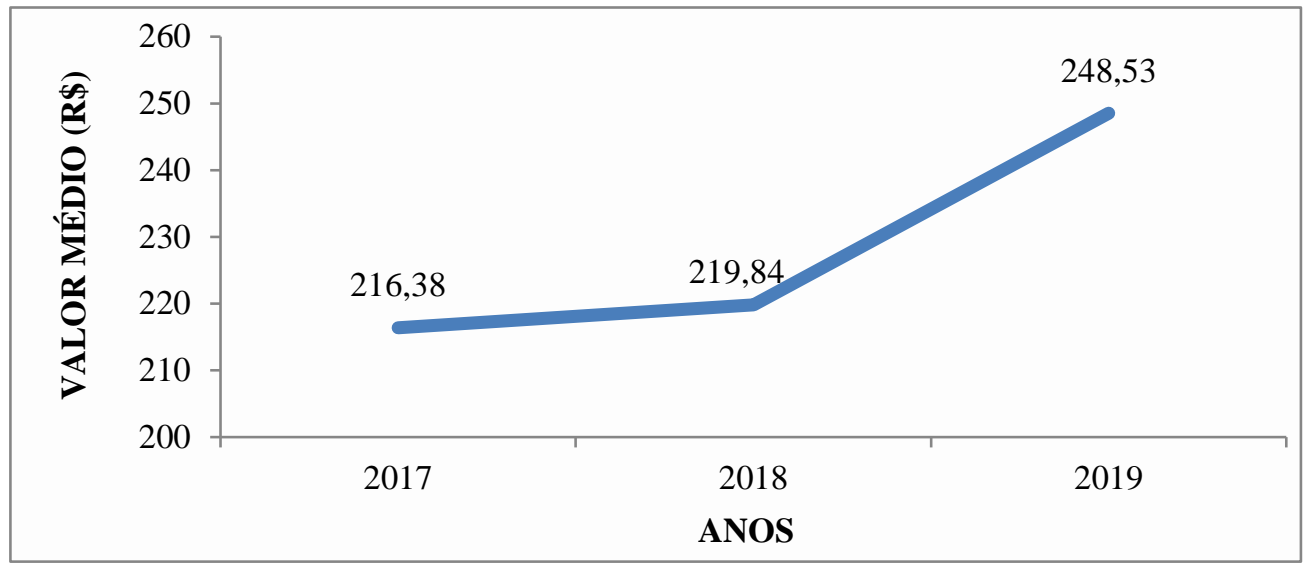

Fonte: DATASUS (2020).

No gráfico acima é possível verificar que o valor médio das internações hospitalares após aborto espontâneo variou entre $\mathrm{R}$ \$ 216,38 a R\$ 248, 53, com média de R\$ 228,25 por internação. Entre os anos de 2017 a 2019 houve um pequeno aumento no custo médio das internações. Apesar de não haver um aumento significativo, observa-se que o ápice foi no ano de 2019, o qual registrou uma média de R\$ 32,15 a mais em relação ao ano de 2017.

\section{Discussão}

Segundo Ribeiro et al. (2017) tratando-se do atendimento, o número de internações na rede pública de saúde totalizou 92\% dos atendimentos, comparado a $8 \%$ dos atendimentos nos serviços privados de saúde. A partir desses dados, pode-se observar que houve uma prevalência de atendimento de abortos espontâneos dentro do serviço público de saúde, pois o mesmo possui uma maior acessibilidade socioeconômica.

De acordo com os dados apresentados nesse estudo, o ano de 2017 registrou um total de 1.084 abortos espontâneos. Em 2018, esse número chegou a 1.064, tendo em 2019 um declínio de, aproximadamente, 1,50\% no número de abortos espontâneos. Desses valores, 2.679 casos são de curetagens uterinas realizadas, incluindo todos os tipos de aborto.

Em concordância com a literatura, tem-se a curetagem uterina como o terceiro procedimento obstétrico mais realizado no Sistema Único de Saúde (Brasil, 2011). Conforme Saciloto et al. (2011) a maior permanência hospitalar está ligada a necessidade do anestesiologista, centro cirúrgico disponível e ao aumento dos riscos de infecções durante o esvaziamento da cavidade intrauterina, visto que, as pacientes estão mais expostas durante a realização.

Nesse contexto, a equipe multidisciplinar necessita de profissionais que proporcionem uma assistência humanizada e qualificada a mulher hospitalizada (Strefling, Filho, Demori, Soares MC \& Santos, 2011). Para isso, devem-se desenvolver estratégias que viabilizem fortalecer recursos para enfrentamento e desenvolva intervenções fundamentadas em fontes de apoio para as mães se adaptarem à perda do filho (Rossman, Greene, Kratovil \& Paula, 2017).

A cidade de Fortaleza - Ceará registrou no ano de 2017, um custo médio de $\mathrm{R} \$ 184,89$ referente à realização da curetagem uterina no pós-abortamento, obtidos a partir da amostra com 119 mulheres. Somado a isso, mediante estudos de Lima et al. (2020) o tempo de permanência hospitalar variou de um a sete dias, com média de 1,58 dias. Comparando-se com o presente estudo, em 2017 no Estado de Sergipe, a média de internações registrou 1,48 dias com custos de R \$216,38. Desde 
então, até 2019, essa média aumentou para 1,59 dias e custo médio de R\$248,53.

A partir da observação nos registros do DATASUS, as mulheres com faixa etária entre 20 a 29 anos, apresentam a maior concentração de aborto realizado, seus percentuais variam de $51 \%$ a $82 \%$ do total de mulheres, por existir maior probabilidade de mulheres nessa idade serem casadas, ter vida sexual ativa e estarem em período fértil, resultando em alto índice de gravidez (Brasil, 2009). A instabilidade na relação conjugal é um dos fatores de risco para o abortamento quando observada as variáveis socioeconômicas.

Os dados expostos no presente artigo não apresentaram uma diferença estatisticamente significativa entre os números de abortos espontâneos e curetagens uterinas realizadas no pós-abortamento, tornando-se muito incidente no serviço público de saúde. Com os dados obtidos nas pesquisas de Araújo et al. (2018), pode-se reafirmar que a prevalência dessa prática no tratamento do aborto acontece de forma rotineira e, consequentemente, aumentam o tempo de internação e a exposição aos medicamentos, gerando custos de internações hospitalares para os cofres públicos.

\section{Conclusão}

Desse modo, a literatura científica traz diferentes terapêuticas para o tratamento do aborto espontâneo, destacando a importância da curetagem uterina que, quando executada, o tempo de internação depende de questões socioeconômicas, recursos utilizados, profissionais envolvidos e complicações obstétricas, podendo, a partir dessas variáveis, aumentar a permanência nos centros de saúde e gerar mais custos para os órgãos públicos.

Logo, se percebe a necessidade de capacitar cada vez mais a equipe multiprofissional sobre a prática desse procedimento, por meio de especializações, desenvolvimento das habilidades práticas e atualizações contínuas do conhecimento técnico científico, a fim de prevenir o aparecimento de complicações futuras, amenizando o sofrimento da mulher submetida ao procedimento de curetagem uterina pós-abortamento.

Nota-se a importância desse artigo como estratégia de levantar novos dados referentes aos abortos espontâneos e curetagens uterinas registradas corriqueiramente nos municípios do Estado de Sergipe. Dessarte sugere-se a ampliação da abrangência de pesquisas futuras relacionadas a essa temática no intuito de agregar mais evidências relativas a esse procedimento, como também avaliar as características sociodemográficas das mulheres submetidas a esse tratamento no pósabortamento, podendo assim identificar se existem fatores sociais e demográficos predisponentes, bem como fortalecer o direcionamento das políticas públicas assistenciais.

\section{Referências}

Adesse, L., Jannotti, C. B., Silva, K. S. D., \& Fonseca, V. M. (2016). Abortion and stigma: an analysis of the scientific literature on the theme. Ciencia \& saude coletiva, 21(12), 3819-3832.

Araújo, C. P., Rezende Dornelas, A. C. V., \& Sousa, A. M. (2018). Abordagem terapêutica no processo de esvaziamento uterino. Revista Baiana de Enfermagem32,.

Cardoso, B. B., Vieira, F. M. D. S. B., \& Saraceni, V. (2020). Aborto no Brasil: o que dizem os dados oficiais?. Cadernos de Saúde Pública, 36 , e0188718.

Chaves, G. K., Pereira, L. D. N., \& Sturza, J. M. (2020). A (des) criminalização do aborto: uma questão de saúde pública. Revista Derecho y Salud, (4), 129135 .

Decreto-lei $n .2 .848$, de 7 de dezembro de 1940. Código penal. http://www.planalto.gov.br/ccivil_03/decreto-lei/del2848compilado.htm.

Domingues, R. M. S. M., Silva, C. M. F. P. D., Grinsztejn, B. G. J., Moreira, R. I., Derrico, M., Andrade, A. C., \& Veloso, V. G. (2020). Prevalência e fatores associados ao aborto induzido no ingresso em uma coorte de mulheres vivendo com HIV/aids, Rio de Janeiro, Brasil, 1996-2016. Cadernos de Saúde Pública, 36, e00201318.

Domingues, R. M. S. M., Fonseca, S. C., Leal, M. D. C., Aquino, E. M., \& Menezes, G. (2020). Legal abortion in Brazil: systematic review of the scientific production, 2008-2018. Cadernos de Saúde Pública, 36, e00189718.

França, C. P., Sakae, T. M., \& Kleveston, T. (2018). Fatores de risco para abortamento em um hospital de referência no sul do Brasil: um estudo caso- 
Research, Society and Development, v. 10, n. 1, e50410111901, 2021

(CC BY 4.0) | ISSN 2525-3409 | DOI: http://dx.doi.org/10.33448/rsd-v10i1.11901

controle. Arquivos Catarinenses de Medicina, 47(2), 35-48.

Lima, K. J., Pinto, F. J. M., Carvalho, F. H. C., Linard, C. F. B. M., Santos, F. C. R. D., Teófilo, F. K. S., \& Nunes, G. P. (2020). Atenção ao abortamento em instituições hospitalares da rede SUS de Fortaleza, Ceará. Cadernos Saúde Coletiva, (AHEAD).

Löwy, I. (2020). Abortion for fetal anomaly: how to speak about a difficult topic. Cadernos de Saúde Pública, 36 , e00188618.

Madeiro, A. P., \& Rufino, A. C. (2017). Maus-tratos e discriminação na assistência ao aborto provocado: a percepção das mulheres em Teresina, Piauí, Brasil. Ciência \& Saúde Coletiva, 22, 2771-2780.

Ministério da Saúde. (2009). Aborto e saúde pública no Brasil: 20 anos/Ministério da Saúde, Secretaria de Ciência, Tecnologia e Insumos Estratégicos, Departamento de Ciência e Tecnologia. Brasília, DF: Ministério da Saúde, 28-29.

Ministério da Saúde. (2011). Atenção humanizada ao abortamento: norma técnica/Ministério da Saúde, Secretaria de Atenção à Saúde, Área Técnica de Saúde da Mulher: Ministério da Saúde, 29-39.

Pereira A. S. et al. (2018). Metodologia da pesquisa científica. [e-book]. Santa Maria. Ed. UAB/NTE/UFSM. https://repositorio.ufsm.br/bitstream/handle/1/15824/Lic_Computacao_Metodologia-Pesquisa-Cientifica.pdf?sequence=1.

Ribeiro, C. L., Albuquerque, F. D. O., \& Souza, A. R. D. (2017). Internações por aborto espontâneo: um retrato de sua ocorrência em Fortaleza. Enferm. foco (Brasília), 37-41.

Rossman, B., Greene, M. M., Kratovil, A. L., \& Meier, P. P. (2017). Resilience in mothers of very-low-birth-weight infants hospitalized in the NICU. Journal of Obstetric, Gynecologic \& Neonatal Nursing, 46(3), 434-445.

Saciloto, M. P., Konopka, C. K., Velho, M. T. D. C., Jobim, F. C., Resener, E. V., Muradás, R. R., \& Nicolaou, P. K. (2011). Aspiração manual intrauterina no tratamento do abortamento incompleto até 12 semanas gestacionais: uma alternativa à curetagem uterina. Revista Brasileira de Ginecologia e Obstetrícia, 33(10), 292-296.

Silva, L., Sales, N., Santos, R., \& Albuquerque, N. (2020). Percepção das mulheres em situação de Abortamento frente ao cuidado de Enfermagem. Revista Ciência Plural, 6(1), 44-55.

Strefling, I. D. S. S., Lunardi Filho, W. D., Demori, C. C., Soares, M. C., \& dos Santos, C. P. (2015). Cuidado de enfermagem à mulher em situação de aborto: revisão integrativa. Revista de Enfermagem da UFSM, 5(1), 169-177. 